\title{
The Effect of Meditation on Concentration Level and Cognitive Performance
}

\author{
Runnan Zhang ${ }^{1}$ \\ ${ }^{1}$ High School, Hyde Park High School, Austin, United States of America \\ Correspondence: Runnan Zhang, Hyde Park High School, Austin, TX 78759, United States of America. E-mail: \\ johnzrn007@gmail.com
}

Received: October 16, 2018 Accepted: December 3, 2018 Online Published: December 23, 2018

doi:10.5539/gjhs.v11n1p134 URL: https://doi.org/10.5539/gjhs.v11n1p134

\begin{abstract}
Many research studies have found that long-duration meditation sessions - ones that last at least 40 minutes a day - can contribute to individuals' concentration and cognitive performance. However, the effects of comparatively brief meditation sessions have not been widely studied. In this study, we tested whether a short meditation session affects cognitive performance and concentration when compared to a control group. Participants with no prior meditation experience underwent a short meditation session lasting 10 minutes, and then completed a section of either the reading or math portion of the SAT. We find no evidence that the short meditation session improves test performance, but subjective feedback from the participants suggests that meditation has a positive impact on their concentration level. The implications of this study and possibilities for future research on the topic will be discussed further.
\end{abstract}

Keywords: concentration level, cognitive performance, meditation

\section{History of the Study of Meditation}

Meditation can be practiced in different ways that vary considerably across cultures and religions, though it originated from Buddhist meditation traditions. Meditation in its various forms has been applied to support multiple mental and physical health conditions since the 1990s, and has also received much attention in psychological research. Currently, meditation is typically described as a practice of non-judgmental attention to the experience in the present moment. (Hart, 2012) This definition states that meditation requires both the regulation of attention (in order to maintain focus on immediate experiences, such as thoughts, emotions, body posture and sensations) and the ability to approach one's experience and environment with openness and acceptance. (Tang, Holzel, \& Posner, 2015)

Meditation has also been the subject of neuroscientific research and healing practices. There are programs such as mindfulness-based stress reduction (MBSR) and integrative body-mind training (IBMT) that use mindfulness meditation as their basis. Evidence suggests that meditation can enhance individuals' self-regulation, which includes attention control, emotional regulation, and self-awareness. The attention network test (ANT) and other experimental paradigms have been used to investigate the effects of meditation on attention performance. (Chiesa, Calati, \& Serretti, 2011)

\section{Introduction}

In "The Real Meaning of Meditation," Swami Rama writes: "Meditation is a precise technique for resting the mind and attaining a state of consciousness that is totally different from the normal waking state...In meditation, the mind is clear, relaxed, and inwardly focused." (Rama, 2013) Along with various other benefits, mindfulness meditation can improve individuals' error detection as well as their attention control. People who meditate are better able to sustain their attention during long tasks.(Tang et al., 2015) Research has shown that after three months of intensive meditation training ten to twelve hours a day, individuals are better able to sustain their attention during a dichotic listening test. (Brefczynski-Lewis, Lutz, Schaefer, Levinson, \& Davidson, 2007) In addition, empirical evidence indicates that meditation training lasting only four days can enhance individuals' ability to sustain attention - benefits that are similar to those of long-term meditation training. (Zeidan, Johnson, Diamond, David, \& Goolkasian, 2010)

Someone practicing mindfulness meditation focuses on the sensation of breath and body -- the air entering and 
leaving their nostrils and the environment around their body -- while maintaining a considerably relaxed state. During the practice of meditation, the meditator learns to acknowledge their thoughts and return his/her attention back to their breaths.

Besides enhancing individuals' attention during a task, meditation can also improve individuals' cognitive performance, as many studies have demonstrated. The N-back task, a task in which participants have to temporarily memorize information from the past then recall the information at the present, (Farnsworth, 2016) can determine individuals' cognitive performance. Through the multivariate analysis of variance (MANOVA) on the scores from the N-back task, a study showed significant improvements on scores and accuracy after meditation. The subjects who practiced meditation over four weeks out-performed a control group in multiple tests that measures cognitive abilities. In a verbal fluency test and the N-back task, (Farnsworth, 2016) the subjects prepared with long-term, long-duration meditation sessions were better able to perform better on cognitive tasks, increase attention, and relieve stress. It is possible that the calming effects of mindfulness meditation combined with the increased capacity to focus on the present improved cognitive performance after meditation training. Individuals who meditated were able to maintain focus and accurately retrieve information from working under conditions that require more rapid stimulus processing. (Tang et al., 2015) Many studies have shown that meditation improves conflict monitoring, the ability to detect errors, which contributes to individuals' cognitive performance. For example, a longitudinal study showed that only 5 days of integrative body-mind training (IBMT) 20 minutes per day led to improved conflict monitoring. (Tang et al., 2015)

Although research studies examining the effects of long- and short-term meditation interventions are growing, the effects of an extremely brief meditation session remain relatively unknown. In the present study, we examined whether a 10-minute meditation session can impact the subject's concentration level, to be measured through a task following their meditation. Specifically, we examined whether meditation can affect an individual's performance on the SAT math or reading test.

\section{Muse Headband and EEG}

The study required a standardized means of measuring the subjects' cognitive performance and concentration. For this we used a device adapted from the electroencephalogram (EEG), or the depiction of the electrical activity occurring at the surface of the brain; this activity appears on the screen of the EEG machine. Waveforms of varying frequency and amplitude measured in microvoltage EEG waveforms are generally classified according to their frequency, amplitude, and shape, as well as the sites on the scalp at which they are recorded (see Figure 1). The classifications of EEG frequencies usually include alpha, beta, theta, and delta.(Sucholeiki, 2017) The data is collected from the brain using electrode sensors on the scalp.

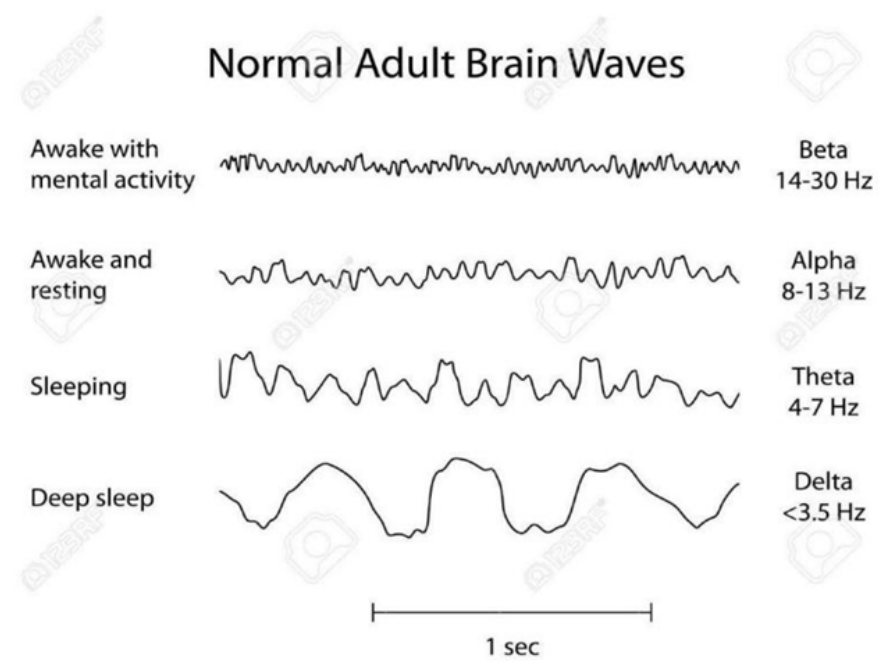

Figure 1. Image showing normal brain wave patterns

The electrical impulses collected from the brain are constant, and vary based on the subject's state of mind, as illustrated above. To describe brain waves, voltage and frequency need to be collected. The voltage of the neurons ranges from 0 to 200 microvolts; the frequency ranges from 1 to 50 times per second. 


\begin{tabular}{lll} 
Wave & Frequency & Comments \\
\hline Beta $(\beta)$ & High & $\beta$ waves are typical of awake, mentally active individuals (with eyes open) \\
\hline Alpha $(\alpha)$ & Low & $\alpha$ waves are seen in awake individuals (with eyes closed) \\
\hline Theta $(\theta)$ & Low & $\theta$ waves are seen in drowsy or very relaxed states \\
\hline Delta $(\delta)$ & Low & $\begin{array}{c}\delta \text { waves are seen in deep sleep when the cortex and thalamus are highly } \\
\text { synchronized }\end{array}$
\end{tabular}

Figure 2. Image showing brain wave frequencies and meanings

Beta waves dominate when people are hyper-alert or anxious. Alpha waves appear when people close their eyes and relax, and disappear when people open their eyes or become more alert in their thinking. Theta waves appear when people are drowsy or not fully paying attention. Delta waves appear when people are resting or sleeping (Figure 2). (Sivakumar, 2017)

The Muse headband is a device that has 7 sensors to detect brain waves. It transfers the data collected via Bluetooth to the "smart" device (see Figure 3). The Muse app offers meditation lessons that help users to meditate better, and it also features real-time feedback. When a person's mind is calm, the sound of the weather is nice; when they let their thoughts wander, the weather gets more intense. After each meditation session feedback is provided for the entire meditation session, which shows when the brain was in calm, neutral, and active states during the meditation. (Muse, 2018)

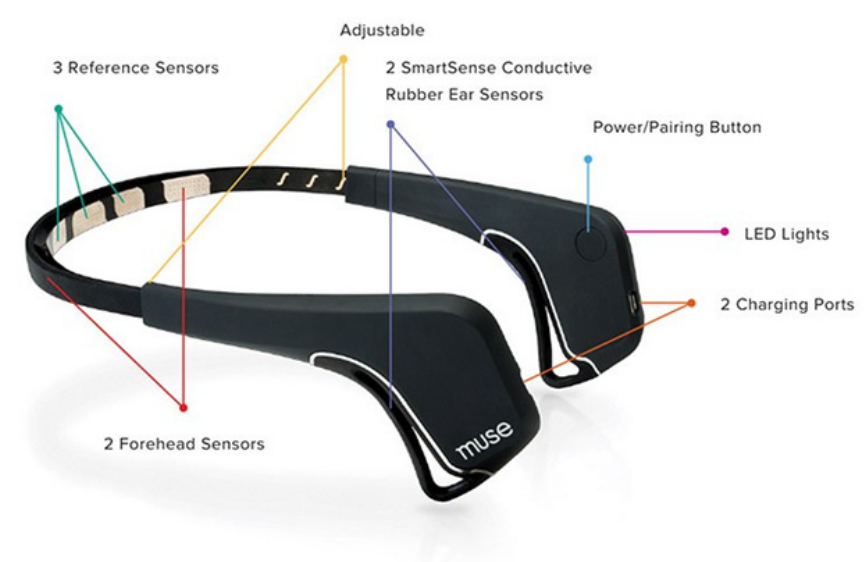

Figure 3. Muse Headband Sensors

\section{Methods}

\subsection{Participants}

The participants included six Chinese students studying in China and the US. The participants were four males and two females, all of whom were born in Qingdao, Shandong. With parental consent, we acquired participants whose ages ranging from 15 to 17, and all were enrolled in either a Chinese public high school or a private school in the United States. None of the participants had any prior meditation experience.

\subsection{Materials}

We used questions from the SAT Official Study Guide as a measure of cognitive performance, and the subjects could use a graphing calculator for the math portion. We also implemented the Muse headband to collect the raw EEG data. The participants listened first to the brief voice introduction to meditation with simple instructions from the Muse app on the iPhone.

\subsection{Design}

We conducted a 2 X 2 factorial designed experiment. This included two independent variables each with two levels (i.e. a temperature of 80 degrees versus a temperature of 40 degrees), with which we investigated the effects of 
meditation (10-minutes meditation versus no meditation) and test categories (math or reading) on the subjects' cognitive performance and concentration level.

\subsection{Procedures}

The test category, math or reading, was chosen at random, and each participant takes two tests of the same category; between the two tests participants were given a 30-minute break. Participants completed a 10-minute meditation before either the first test or the second test, and the meditation was accompanied by a brief voice guide that explained the basics of how meditation works. Participants completed the test in a quiet environment. The questions were chosen from the SAT Official Study Guide, along with an answer sheet. After each test, the participants are asked how concentrated they felt on a scale from one to ten, and they provided comments about their testing experience.

\section{Results}

To analyze the collected data and examine the effect of a brief meditation training on participants' cognitive performance and concentration, we used an ANOVA (analysis of variance) on the data using SPSS Statistics (Statistical Package for the Social Sciences) by MIT. The accuracy of each test is recorded as a percentage, and the participants' self-rated concentration level is also recorded.

Table 1. ANOVA analysis using SPSS on scores

Descriptive statistics

Dependent variable: score percentage

\begin{tabular}{lllll}
\hline Type & Meditation & Mean & Std. Deviation & N \\
\hline \multirow{3}{*}{ Reading } & No meditation & 69.6438 & 10.57780 & 8 \\
& Meditation & 71.8025 & 8.93838 & 8 \\
& Total & 70.7231 & 9.52586 & 16 \\
Math & No meditation & 83.3350 & 23.03503 & 6 \\
& Meditation & 83.3350 & 23.03503 & 6 \\
\hline \multirow{2}{*}{ Total } & Total & 83.3350 & 21.96304 & 12 \\
& No meditation & 75.5114 & 17.71349 & 14 \\
\hline
\end{tabular}

Test of Between-Subjects Effects

Dependent variable: score percentage

\begin{tabular}{llllll}
\hline Source & Type III Sum of Squares & df & Mean Square & F & Sig. \\
\hline Corrected Model & $1109.334^{\mathrm{a}}$ & 3 & 369.778 & 1.335 & .286 \\
Intercept & 162746.783 & 1 & 162746.783 & 587.479 & .000 \\
Type & 1090.693 & 1 & 1090.693 & 3.937 & .059 \\
Meditation & 7.989 & 1 & 7.989 & .029 & .867 \\
Type* Meditation & 7.989 & 1 & 7.989 & .029 & .867 \\
Error & 6648.617 & 24 & 277.026 & 12 & \\
Total & 170032.091 & 28 & & & \\
Corrected Total & 7757.951 & 27 & & & \\
\hline
\end{tabular}

Table 1 represents a 2 (types of test -- math and reading) x 2 (meditation/ no meditation) ANOVA analysis. The statistical result showed marginal effects for the type of test but no significant result over meditation. The result [Graph 1] shows that the math average $(\mathrm{M}($ mean $)=83.34, \mathrm{SD}($ standard deviation $)=21.96)$ is $13.6 \%$ higher than 
the reading average $(\mathrm{M}=70.72, \mathrm{SD}=9.53)$. This difference was marginally significant: $\mathrm{F}$-value (to reject the null-hypothesis, the f-value needs to be closer to 1 .) $(1,24)=3.94, \mathrm{p}$ (the statistical significance of the data) $=0.06$. The statistical result is significant when $\mathrm{p}$ is less than 0.06 . The test score average of participants who meditated before the test (math and reading) $(\mathrm{M}=76.75, \mathrm{SD}=16.80)$ proved to be $1.23 \%$ more accurate than the control group $(\mathrm{M}=75.51, \mathrm{SD}=17.71)$. This difference was not significant: $\mathrm{F}(1,24)=0.03, \mathrm{p}=.87$. The interaction effect between the type and meditation was not significant either: $\mathrm{F}(1,24)=0.03, \mathrm{p}=.87$.

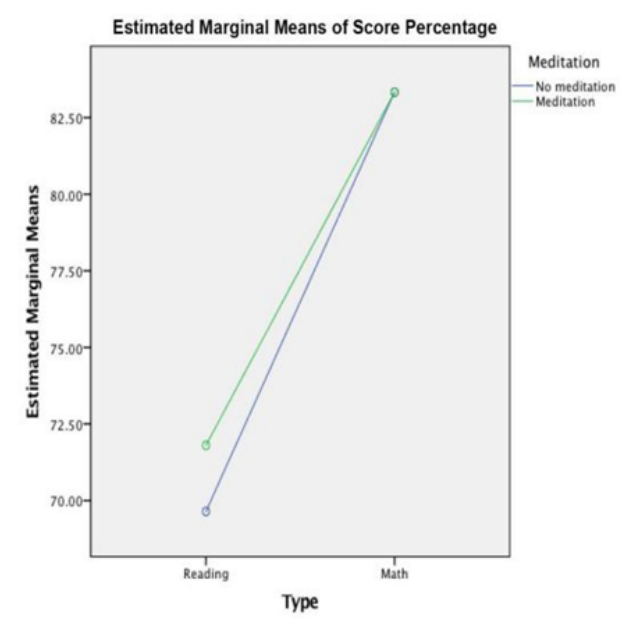

Graph 1. Score Average of Type of Test and Meditation

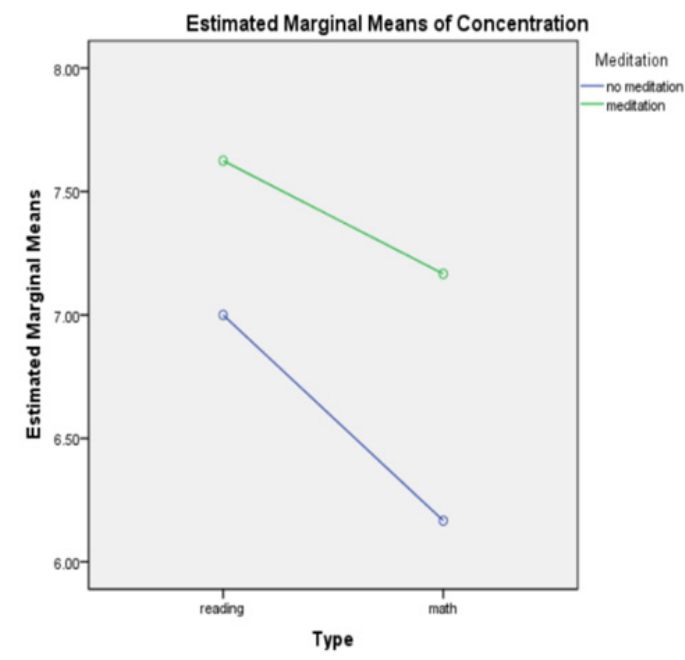

Graph 2. Estimated Marginal Means of Rated Concentration

We also did an ANOVA on the participants' self-scored concentration (Table 2). The rating of the tests after meditation $(\mathrm{M}=7.4286, \mathrm{SD}=1.65084)$ is higher than the tests without meditation, but the result was not statistically significant: $F(1,24)=1.40, p=.25$. As Graph 2 shows, the participants reported higher concentration during the reading test $(\mathrm{M}=7.31, \mathrm{SD}=1.14)$ than the math test $(\mathrm{M}=6.67, \mathrm{SD}=2.239)$, but the ANOVA analysis (Table 3) shows no significance in the results. 
Table 2. Descriptive Statistics of Mean and Standard Deviation

Dependent variable:Concentration

\begin{tabular}{lllll}
\hline Type & Meditation & Mean & Std. Deviation & N \\
\hline \multirow{3}{*}{ Reading } & No meditation & 7.000 & 1.30931 & 8 \\
& Meditation & 7.6250 & .91613 & 8 \\
\hline \multirow{3}{*}{ Math } & Total & 7.3125 & 1.13835 & 16 \\
& No meditation & 6.1667 & 2.48328 & 6 \\
\hline \multirow{3}{*}{ Total } & Meditation & 7.1667 & 2.40139 & 6 \\
& Total & 6.6667 & 2.38683 & 12 \\
& No meditation & 6.6429 & 1.86495 & 14 \\
\hline
\end{tabular}

Table 3. ANOVA analysis on Rated Concentration level

Dependent variable: Concentration

\begin{tabular}{llllll}
\hline Source & Type III Sum of Squares & df & Mean Square & F & Sig. \\
\hline Corrected Model & $7.423^{\mathrm{a}}$ & 3 & 2.474 & .766 & .524 \\
Intercept & 1340.003 & 1 & 1340.003 & 414.746 & .000 \\
Type & 2.860 & 1 & 2.860 & .885 & .356 \\
Meditation & 4.527 & 1 & 4.527 & 1.401 & .248 \\
Type* Meditation & .241 & 1 & .241 & .075 & .787 \\
Error & 77.542 & 24 & 3.231 & & \\
Total & 1471.000 & 28 & & & \\
Corrected Total & 84.964 & 27 & & & \\
\hline
\end{tabular}

Participants left a simple comment about their experience with meditation after the test. Their responses (see Figure 4) demonstrated the positive impact on their test experience. Participant 2, for example, noted improvement in their concentration after meditation.

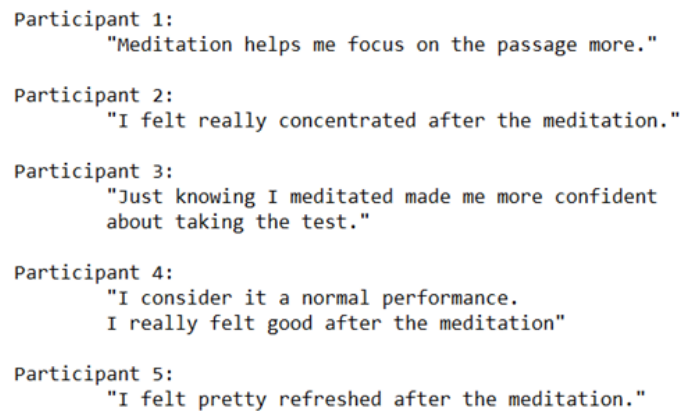

Figure 4. Participants' responses after the test with meditation

\section{Discussion of Results}

We found that the brief meditation session did not have a significant impact on participants' cognitive performance. However, the participants' survey demonstrated that meditation has a positive impact on their concentration from a subjective, though not empirical, standpoint. For example, one participant noted, "I felt pretty refreshed after the meditation." Others reported, "I think meditation helps me focus on the passage more." Another participant stated: "Just knowing I meditated made me more confident about taking the test." It is also important to reiterate that all of 
the participants never had prior meditation experience. This implies that further study into a longitudinal research on effects of meditation may offer important insights.

\section{Suggestions for Future Study}

Future research should conduct similar studies with larger samples. Given that the sample size in this study was limited, its findings were also limited in respect to statistical accuracy. Future work should use tests that are adequately challenging to better examine how meditation can support students' performance in cognitive tasks. We could also conduct a more individually-designed study in which the tests are fit to each participants' aptitude in reading or math. Future studies may also include longer meditation sessions (i.e. 20-30 minutes) with more detailed instructions, because the introduction that the Muse app provided might be too concise to ensure participants had a comprehensive understanding of meditation. One participant even mentioned that longer instructions and more tips would help to improve his meditation experience.

If future studies were to prove that meditation helps people improve their cognitive abilities and concentration, the impact on the education community would be immense. Students could easily meditate before their tests and enjoy greater success. Meditation would be a tool employed by many students to facilitate their learning experience and allow them to work more efficiently. Moreover, imagine what an impact meditation would have on office workers with heavy cognitive tasks. They could perform better with meditation and be more focused during meetings to increase their productivity. It is clear that the successful application of meditation could bring many benefits to our world.

\section{Competing Interests Statement}

The authors declare that there are no competing or potential conflicts of interest.

\section{References}

Brefczynski-Lewis, J. A., Lutz, A., Schaefer, H. S., Levinson, D. B., \& Davidson, R. J. (2007). Neural correlates of attentional expertise in long-term meditation practitioners. Proc Natl Acad Sci U S A, 104(27), 11483-11488. https://doi.org/10.1073/pnas.0606552104

Chiesa, A., Calati, R., \& Serretti, A. (2011). Does mindfulness training improve cognitive abilities? A systematic review of neuropsychological findings. Clin Psychol Rev, 31(3), 449-464. https://doi.org/10.1016/j.cpr.2010.11.003

Farnsworth, B. (2016). N-back to Basics: Learning and Momory with the N-back Test. Retrieved from https://imotions.com/blog/n-back-test/

Hart, W. (2012). The art of living: Vipassana meditation as taught by S.N. Goenka. Maharashtra, India: Vipassana Research Institute.

Muse. (2018). What it Measures. Retrieved from https://choosemuse.com/what-it-measures/

Rama, S. (2013). The Real Meaning of Meditation. Retrieved from https://yogainternational.com/article/view/the-real-meaning-of-meditation

Sivakumar, S. (2017). The Wave - The characteristics of an EEG. Retrieved from https://www.firstclassmed.com/articles/2017/eeg-waves

Sucholeiki, R. (2017, Oct 06, 2017). Normal EEG Waveforms. Retrieved from https://emedicine.medscape.com/article/1139332-overview\#a1

Tang, Y. Y., Holzel, B. K., \& Posner, M. I. (2015). The neuroscience of mindfulness meditation. Nat Rev Neurosci, 16(4), 213-225. https://doi.org/10.1038/nrn3916

Zeidan, F., Johnson, S. K., Diamond, B. J., David, Z., \& Goolkasian, P. (2010). Mindfulness meditation improves cognition: evidence of brief mental training. Conscious Cogn, 19(2), 597-605. https://doi.org/10.1016/j.concog.2010.03.014

\section{Copyrights}

Copyright for this article is retained by the author(s), with first publication rights granted to the journal.

This is an open-access article distributed under the terms and conditions of the Creative Commons Attribution license (http://creativecommons.org/licenses/by/4.0/). 